\section{Monopolar Transurethral Resection of Prostate for Benign Prostatic Hyperplasia in Patients With and Without Preoperative Urinary Catheterization: A Prospective Comparative Study}

Baikuntha Adhikari ${ }^{1}$, Anil Shrestha ${ }^{1}$, Robin B. Basnet ${ }^{1}$, Parash M. Shrestha ${ }^{1}$, Binod B. Gharti ${ }^{1}$, Arvind K. Shah ${ }^{1}$

1. Urology, National Academy of Medical Sciences, Bir Hospital, Kathmandu, NPL

Corresponding author: Baikuntha Adhikari, baikuntha2040@gmail.com

\section{Abstract}

\section{Background}

A significant proportion of patients undergo surgery for benign prostatic hyperplasia following acute urinary retention. Studies have reported conflicting results of improvement following transurethral surgery in these patients.

\section{Objective}

To compare perioperative complications and postoperative voiding parameters in patients undergoing monopolar transurethral resection of prostate with and without preoperative Foley catheterization.

\section{Methods}

A prospective non-randomized study was conducted in patients undergoing monopolar transurethral resection of prostate for symptomatic benign prostatic hyperplasia. Patients were divided into those with Foley catheterization preoperatively $(n=52)$, and those without catheters $(n=90)$. Change in hemoglobin level, the resected volume of prostate, complications and the need for postoperative catheterization were compared. Postoperative symptoms score using International Prostate Symptom Score, maximum flow rate and post-void residual volume were assessed at three months follow up.

\section{Results}

The mean operative duration, length of stay and resected volume were higher in those patients with catheters; however, no significant differences were noted for mean hemoglobin level change and need for postoperative recatheterization. Three patients in each group required recatheterization and, all were catheter-free at one week postoperatively. Complications developed in $16.1 \%(n=23)$ with most of them being Clavien I. Patients with catheters had a lower postoperative maximum flow rate than those without it ( $16.90 \mathrm{vs} 19.75 \mathrm{~mL} / \mathrm{sec})$. Patients with catheters had a significantly better postoperative quality of life and symptom score.

\section{Conclusion}

Monopolar transurethral resection of prostate in patients with preoperative per-urethral Foley catheter for acute urinary retention had similar postoperative voiding parameters with comparable complication rates to those without a catheter.

Categories: Urology

Keywords: acute urinary retention, benign prostatic hyperplasia, monopolar, transurethral resection of prostate, trial without catheter

\section{Introduction}

Benign prostatic hyperplasia (BPH) prevalence increases with age and affects nearly three-quarters of men by the seventh decade of life $[1,2]$. Acute urinary retention (AUR) is the predominant complaint in a significant proportion of patients (20\%-42\%) undergoing surgery for BPH [3-5]. Even with the availability of various transurethral procedures, monopolar transurethral resection of prostate (TURP) is still considered the reference standard surgical treatment of BPH for the prostate size of 30-80 mL and continues to be one of the most frequently performed procedures in urological practice [6-10].

Higher incidence of perioperative complications, poor postoperative voiding outcomes and increased postoperative recatheterization rates have been documented in patients undergoing monopolar TURP in 
patients with a history of AUR [11,12]. In view of these findings, patients with AUR were counseled regarding the higher risk of perioperative morbidity. In contrast, recent studies have shown a reduced complications rate along with the reduced rate of recatheterization with similar voiding parameters postoperatively in these patients $[13,14]$.

In our part of the world, patients have preoperative Foley catheterization for a longer duration due to limited availability of urological care outside the major cities, financial constraints and even fear of surgery, in addition to the limited slots available for surgery in public hospitals $[5,15]$. There are limited studies addressing both the objective and subjective voiding outcomes of TURP among catheterized patients after failure of at least one trial without catheter (TWOC). Hence, this study was undertaken to compare outcomes of TURP between patients with per-urethral Foley catheter preoperatively after failed TWOC and those with no preoperative Foley catheterization. The outcome of this study can be useful in counseling patients with catheters preoperatively.

\section{Materials And Methods}

This prospective comparative study was conducted in patients undergoing TURP for the period of 18 months from March 2019 to August 2020 in the Department of Urology of Bir Hospital, Kathmandu, Nepal. Ethical clearance was obtained from the Institutional Review Board of the National Academy of Medical Sciences (Ref. no. 529/077/78). Informed written consent was obtained from all patients.

Patients requiring TURP were divided into two groups, those with preoperative per-urethral Foley catheter after the failure of at least one TWOC (Group 1) and those with moderate to severe bothersome LUTS but no Foley catheter preoperatively (Group 2). Patients with a history of prostate surgery, prostate cancer, bladder cancer, urethral stricture surgery, the concurrent presence of urinary bladder stone, urethral stricture, neurogenic bladder and the presence of large urinary bladder diverticula were excluded.

Patients were assessed preoperatively performing general physical examination including digital rectal examination and brief neurological examination. The patient's age, body mass index (BMI), American Society of Anesthesiologists' classification (ASA) and prostate-specific antigen (PSA) level were recorded. Group 2 underwent preoperative uroflowmetry to document maximum flow rate (Qmax) and flow pattern, together with post-void residual (PVR) volume, and International Prostate Symptom Score (IPSS) was obtained. All the patients underwent transabdominal ultrasound to document the prostate size and intravesical prostatic protrusion. In Group 1 patients were offered at least a trial to self-void after removal of Foley Catheter before posting for surgery and the duration of Foley catheterization and the previous number of TWOC attempts were documented.

Patients with a positive urine culture were treated with appropriate antibiotics for seven days, following which urine culture was repeated. Patients with repeat culture positivity were admitted and treated with an injectable antibiotic for three days prior to surgery. All the surgeries were done under spinal anesthesia by four experienced urologists. All patients received prophylactic Ceftriaxone 1gm at the time of anesthesia. TURP was performed using 26 French rotating continuous flow monopolar resectoscopes with standard monopolar loop (Karl Storz, Tuttlingen, Germany) and generator Force FX (Medtronic, Minneapolis, MN, USA) set at 130 Watt pure cut and 80 Watt spray coagulation mode. Glycine (1.5\%) was used as the irrigation fluid. At the completion of TURP, 22 French three-way Foley catheter was inserted per urethra and inflated with $30 \mathrm{~mL}$ distilled water. Continuous bladder irrigation was commenced with a normal saline solution which was maintained for 24 hours. Resected tissue was weighed using a digital weighing machine and was submitted for histopathological assessment. The catheter was removed on the second postoperative day. Patients were discharged on the same day once they voided twice after removal of the catheters. On failure of this trial the patients were recatheterized and discharged on the same day with advice to follow up after a week for a trial off the catheter.

Perioperative variables such as duration of surgery, the weight of resected tissue, hemoglobin change, duration of post-operative Foley catheterization, duration of hospital stay, the need for post-operative Foley recatheterization and complications were recorded. The operating time was calculated as the time from the introduction of the resectoscope till the placement of the Foley catheter.

The proportion of prostate resected was calculated as the ratio of the volume resected and the preoperative prostate volume measured under ultrasound. Post-operative hemoglobin level was assessed at 6 hours and 24 hours after the operation. Change in hemoglobin level was obtained as the difference between its preoperative and postoperative value. Serum electrolytes were assessed immediately and 24 hours after the operation. Complications were classified as per the modified Clavien classification system.

Patients were followed up at two weeks in Urology OPD to assess for hematuria, urinary tract infection and review of histopathology report. Qmax, PVR volume, IPSS and complications were reassessed at three months follow up.

Data analysis was done using the Statistical Package for Social Sciences Windows version 23 (SPSS Inc, 
Chicago, IL, USA). Patient demographic and clinical characteristics were compared between the two groups. Baseline characteristics were compared using the Chi-square test for categorical variables and the Student's t-test/Mann Whitney U test for continuous data. Two-tailed tests were used. One-way ANOVA and Kruskal Wallis test were used to compare continuous variables in more than two groups as appropriate. A p-value < 0.05 was considered statistically significant.

\section{Results}

A total of 196 patients underwent TURP during the study period. Fifty-four patients were excluded from the study and 142 patients were included in the final analysis (Figure 1).

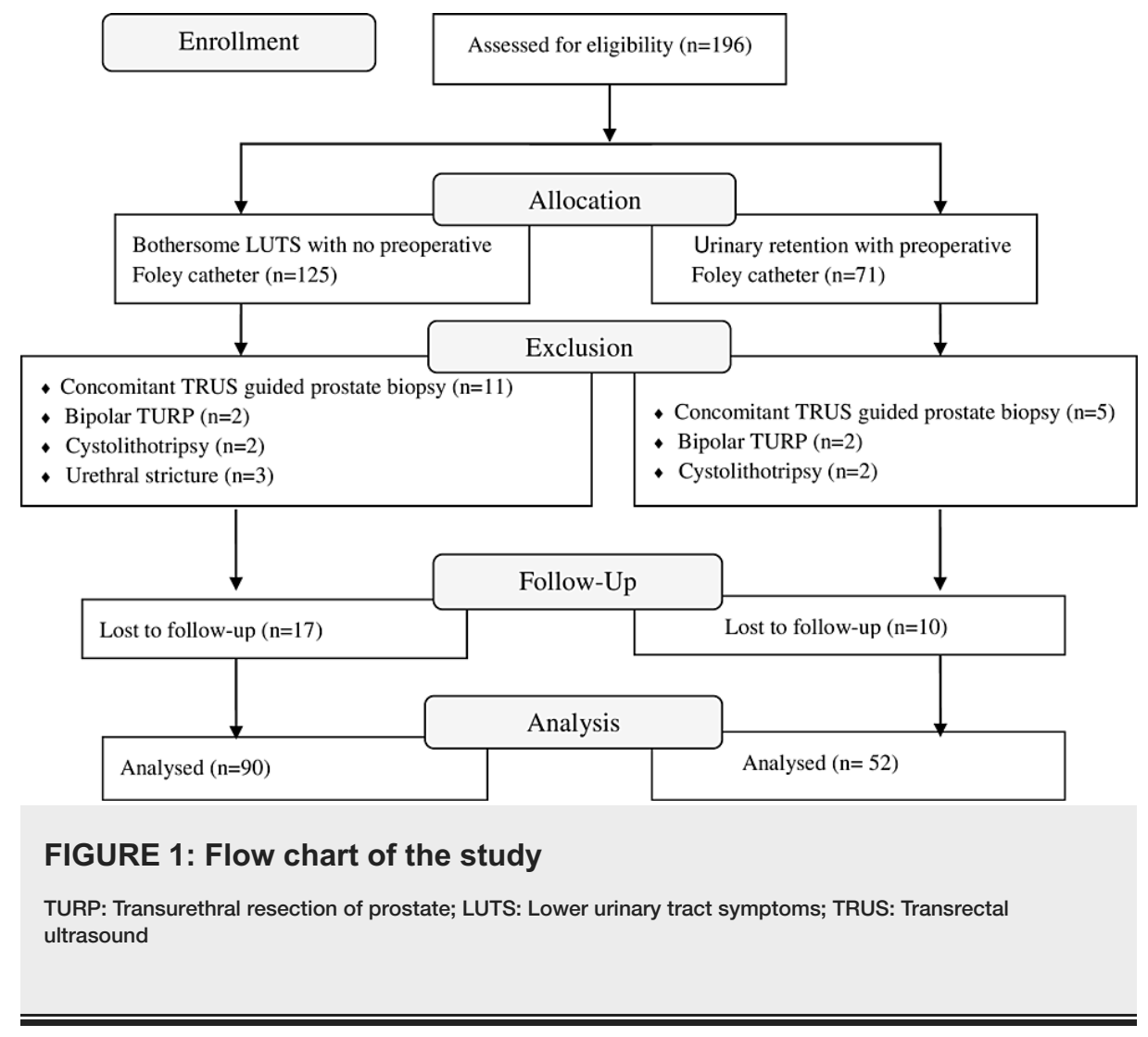

The patients in Group 1 were older than those in Group 2 (70.87 \pm 9.74 vs $66.39 \pm 9.04$ years). PSA level was higher in Group $1(3.87 \pm 2.07 \mathrm{ng} / \mathrm{mL})$ than Group $2(2.77 \pm 1.61 \mathrm{ng} / \mathrm{mL})$, p $<0.001$. The patients without Foley catheters had a higher prevalence of comorbidities (51.1\%) as compared to those with it (28.8\%), $\mathrm{p}=0.01$. Diabetes mellitus, hypertension and chronic airway disease were the common comorbidities in both groups. The mean duration of preoperative Foley catheterization in the catheterized group was 8.25 weeks (range 1 to 32 weeks) (Table 1). The mean prostate volume was higher in Group $1(53.85 \pm 15.90 \mathrm{~mL})$ than in Group 2 $(49.38 \pm 16.19 \mathrm{~mL})$; however, it was not statistically significant $(\mathrm{p}=0.120)$ (Table 1$)$. Preoperative urine culture at any time prior to surgery was positive in 18 (34.6\%) and 4 (4.4\%) patients in Group 1 and Group 2, respectively (Table 1$)$. 


\section{Cureus}

\begin{tabular}{|c|c|c|c|}
\hline Variables & Group1 $(n=52)$ & Group $2(n=90)$ & P-value \\
\hline Age (yrs) & $70.87 \pm 9.74$ & $66.39 \pm 9.04$ & $0.007^{*}$ \\
\hline BMI $\left(\mathrm{kg} / \mathrm{m}^{2}\right)$ & $26.55 \pm 3.29$ & $26.74 \pm 2.43$ & 0.695 \\
\hline ASA Score & & & $0.010^{* *}$ \\
\hline I & $37(71.1 \%)$ & $41(45.5 \%)$ & \\
\hline II & $15(28.8 \%)$ & 47 (52.2\%) & \\
\hline III & $0(0 \%)$ & $2(2.2 \%)$ & \\
\hline \multicolumn{4}{|l|}{ Comorbidities } \\
\hline Yes, n (\%) & $15(28.8 \%)$ & $46(51.1 \%)$ & $0.010^{* *}$ \\
\hline DM (n) & 3 & 10 & \\
\hline $\operatorname{COAD}(\mathrm{n})$ & 4 & 13 & \\
\hline HTN (n) & 6 & 20 & \\
\hline Others (n) & 2 & 3 & \\
\hline Duration of symptoms (months) median (Q1, Q3) & $12(6,24)$ & $18(10,36)$ & $0.207^{\star \star \star}$ \\
\hline IPSS (Total) & NA & $25.58 \pm 5.69$ & NA \\
\hline Storage subscore & NA & $8.55 \pm 3.53$ & NA \\
\hline Voiding subscore & NA & $17.02 \pm 3.17$ & NA \\
\hline QOL score & NA & $5.72 \pm 0.56$ & NA \\
\hline PSA (ng/mL) & $3.87 \pm 2.07$ & $2.77 \pm 1.61$ & $0.001^{*}$ \\
\hline Prostate volume $\left(\mathrm{cm}^{3}\right)$ & $53.85 \pm 15.90$ & $49.38 \pm 16.19$ & 0.113 \\
\hline IVPP (mm) & $15.45 \pm 4.80$ & $13.69 \pm 6.73$ & 0.099 \\
\hline PVR (mL) median (Q1, Q3) & NA & $79.50(40,136)$ & NA \\
\hline Qmax (mL/sec) & NA & $9.20 \pm 3.11$ & NA \\
\hline Urine c/s positive n (\%) & $18(34.61)$ & $4(4.44)$ & $<0.001^{* *}$ \\
\hline
\end{tabular}

\section{TABLE 1: Preoperative characteristics of the study population}

Data presented as mean \pm standard deviation or number (\%) Group 1: with preoperative Foley catheter; Group 2: without Foley catheter.

${ }^{*}$ Student's t-test ${ }^{\star \star}$ Chi-square test ${ }^{* \star *}$ Mann-Whitney U test

ASA: American Society of Anesthesiologists; BMI: Body mass index; COAD: Chronic obstructive airway disease; c/s: culture and sensitivity; DM: Diabetes mellitus; HTN: Hypertension; IVPP: Intravesical prostatic protrusion; IPSS: International Prostate Symptom Score; $\mathrm{mL}$ : milliliter; mm: millimeter; mths: months; NA: Not applicable; PSA: Prostate-specific antigen; PVR: Post-void residual; Q1: First quartile; Q3: Third quartile; Qmax: maximum urinary flow rate; QOL: Quality of life; yrs: years. 


\section{Cureus}

\begin{tabular}{|c|c|c|c|}
\hline Variables & Group $1(n=52)$ & Group $2(n=90)$ & P-value \\
\hline Operation duration (min) & $54.52 \pm 16.48$ & $44.52 \pm 15.24$ & $<0.001^{*}$ \\
\hline Need of active urethral dilatation & $13(25 \%)$ & $37(41.1 \%)$ & $0.053^{\star *}$ \\
\hline Resection weight (gm) & $27.87 \pm 10.90$ & $23.14 \pm 8.67$ & $0.005^{\star}$ \\
\hline Proportion resected (\%) & $51.27 \pm 11.92$ & $47.59 \pm 11.61$ & 0.074 \\
\hline $\mathrm{Hb}$ change $(\mathrm{g} / \mathrm{dL})$ & $1.40 \pm 1.12$ & $1.76 \pm 1.24$ & 0.093 \\
\hline Catheter time (days) & $2.46 \pm 1.09$ & $2.16 \pm 0.77$ & 0.054 \\
\hline Hospital stays (days) & $3.76 \pm 1.24$ & $3.43 \pm 0.76$ & $0.048^{*}$ \\
\hline Catheter free at discharge $\mathrm{n}(\%)$ & $49(90.3 \%)$ & $87(96.6 \%)$ & $0.487^{\star \star}$ \\
\hline
\end{tabular}

\section{TABLE 2: Comparison of perioperative variables between the groups}

Data presented as mean \pm standard deviation and number (percentage).

${ }^{*}$ Student's t-test ${ }^{* *}$ Chi-square test

gm: gram; Hb: Hemoglobin; min: minutes; \%: percent.

The overall complication rate was $16.1 \%$ with no significant difference between the groups $(\mathrm{p}=0.126)$ including those with failure to void after catheter removal. The majority of the complications were Clavien grade I (69.5\%) and Clavien grade II (17.39\%). The overall rate of recatheterization at discharge was $4.2 \%$ with $5.7 \%$ and $3.3 \%$ of patients in Group 1 and Group 2 requiring so respectively. A patient in Group 2 developed transurethral resection syndrome and he had an uneventful recovery after two days of management in the intensive care unit (Table 3). Two patients with catheters (3.8\%) and three patients without catheters (3.3\%) had a urethral stricture.

\begin{tabular}{|c|c|c|c|c|}
\hline Grade & Complications & Group 1 ( $n=14)$ & Group $2(n=9)$ & Management \\
\hline \multirow[t]{4}{*}{ I } & Fever(n) & 2 & 2 & Antipyretic added \\
\hline & Hematuria (n) & 2 & 0 & Prolonged bladder irrigation \\
\hline & Catheter malfunction due to clot (n) & 2 & 2 & Bedside catheter change \\
\hline & Acute retention after Foley removal (n) & 3 & 3 & Foley recatheterization \\
\hline \multirow[t]{2}{*}{ II } & Post op hematuria (n) & 1 & 0 & Transfusion \\
\hline & Fever with signs of bacteremia (n) & 2 & 1 & Antibiotic change \\
\hline IIla & UB clot (n) & 2 & 0 & Cystoscopy and clot evacuation \\
\hline IVb & TUR syndrome (n) & 0 & 1 & Admission in ICU \\
\hline
\end{tabular}

\section{TABLE 3: Comparison of complications between the groups}

ICU: Intensive care unit; TUR: Transurethral resection; UB: Urinary bladder.

Patients in Group 2 demonstrated higher post-operative Qmax than those in Group $1(19.75 \pm 3.12 \mathrm{~mL} / \mathrm{sec}$ vs $16.90 \pm 2.92 \mathrm{~mL} / \mathrm{sec}, \mathrm{p}=0.00$ ), with no difference in PVR volume between the groups (Table 4). The subjective improvement was significantly higher in those with catheters preoperatively as demonstrated by lower mean IPSS and storage symptoms score $(\mathrm{p}=0.013)$ (Table 4). 


\section{Cureus}

\begin{tabular}{|c|c|c|c|}
\hline Variables & Group 1 (n=52) & Group 2 (n=90) & P-value \\
\hline Catheter free at follow up (\%) & 100 & 100 & \\
\hline Qmax (mL/sec) & $16.90 \pm 2.92$ & $19.75 \pm 3.12$ & $<0.001^{*}$ \\
\hline PVR (mL) median (Q1, Q3) & $40(20,50)$ & $25(10,50)$ & $0.922^{\star \star}$ \\
\hline IPSS (Total) & $6.63 \pm 2.68$ & $7.46 \pm 2.22$ & 0.050 \\
\hline Storage subscore & $2.13 \pm 1.64$ & $2.73 \pm 1.35$ & $0.021^{\star}$ \\
\hline Voiding subscore & $4.50 \pm 1.44$ & $4.72 \pm 1.36$ & 0.363 \\
\hline QOL score & $1.23 \pm 0.42$ & $1.44 \pm 0.52$ & $0.013^{*}$ \\
\hline
\end{tabular}

\section{TABLE 4: Comparison of outcome variables at three months follow up}

Data presented as mean \pm standard deviation

Group 1: with preoperative Foley catheter; Group 2: without Foley catheter.

*Student's t-test ${ }^{* *}$ Mann-Whitney U test

IPSS: International Prostate Symptom Score; PVR: Post-void residual; Qmax: maximum urinary flow rate; Q1: First quartile; Q3: Third quartile; QOL: Quality of life.

There was no difference in the preoperative prostate volume and volume of prostate resected during TURP. Postoperative hemoglobin change, duration of Foley catheterization postoperatively, IPSS score and Q max at three months were similar between the groups (Table 5). Among patients with preoperative Foley catheters for more than 12 weeks, complications occurred in three patients and two patients required recatheterization after failure to void postoperatively (Table 5). 


\begin{tabular}{|c|c|c|c|c|c|}
\hline \multirow[b]{2}{*}{ Variables } & \multicolumn{5}{|c|}{ Duration of preoperative perurethral Foley catheterization } \\
\hline & $<4$ weeks $(n=19)$ & 4-8 weeks $(n=15)$ & 8-12 weeks $(n=9)$ & $>12$ weeks $(n=9)$ & P-value \\
\hline Prostate volume $(\mathrm{mL})$ & $50.31 \pm 13.79$ & $57.43 \pm 20.00$ & $55.45 \pm 14.79$ & $53.76 \pm 14.46$ & 0.315 \\
\hline Resected Prostate volume (gm) & $25.15 \pm 9.79$ & $28.10 \pm 12.65$ & $30.16 \pm 10.39$ & $30.94 \pm 10.94$ & $0.008^{\star}$ \\
\hline Resected proportion (\%) & $49.72 \pm 12.35$ & $48.24 \pm 10.37$ & $54.06 \pm 11.79$ & $56.78 \pm 13.02$ & 0.301 \\
\hline $\mathrm{Hb}$ change (g/dL) & $1.28 \pm 1.20$ & $1.62 \pm 1.20$ & $1.65 \pm 0.99$ & $1.04 \pm 0.99$ & 0.561 \\
\hline Postoperative Foley catheter duration (days) & $2.58 \pm 1.30$ & $2.33 \pm 0.61$ & $2.00 \pm 0.00$ & $2.89 \pm 1.61$ & 0.340 \\
\hline PVR at three months $(\mathrm{mL})$ median $(\mathrm{Q} 1, \mathrm{Q} 3)$ & $25(10,50)$ & $45(30,50)$ & $28(10,40)$ & $45(10,55)$ & $0.112^{* *}$ \\
\hline QOL score at three months & $1.36 \pm 0.49$ & $1.26 \pm 0.45$ & $1.11 \pm 0.33$ & $1.00 \pm 0.00$ & 0.139 \\
\hline IPSS at three months & $6.16 \pm 2.93$ & $6.87 \pm 2.85$ & $7.11 \pm 2.20$ & $6.78 \pm 2.58$ & 0.806 \\
\hline Qmax at three months $(\mathrm{mL} / \mathrm{sec})$ & $16.77 \pm 2.46$ & $18.13 \pm 3.50$ & $16.55 \pm 2.00$ & $15.44 \pm 3.08$ & 0.166 \\
\hline Complication (n) & 7 & 2 & 2 & 3 & $0.449^{\star \star \star}$ \\
\hline Foley catheter at discharge (n) & 1 & 0 & 0 & 2 & $0.114^{* * *}$ \\
\hline
\end{tabular}

\section{TABLE 5: Comparison between patients with various duration of Foley catheterization preoperatively}

Data presented as mean \pm standard deviation and numbers

*One-way ANOVA test ${ }^{* \star}$ Kruskal Wallis test ${ }^{* \star \star}$ Chi-square test

gm: gram; g/dL: gram/deciliter; Hb: Hemoglobin; IVPP: Intravesical prostatic protrusion; IPSS: International Prostate Symptom Score; mL: mililiter; PSA: Prostate-specific antigen; PVR: Post-void residual; Q1: First quartile; Q3: Third quartile; Qmax: maximum urinary flow rate; QOL: Quality of life.

\section{Discussion}

A significant number of patients in developing countries still undergo BPH-related surgery for AUR after the failure of TWOC, which is in contrast to western countries where bothersome LUTS accounts for the majority of the cases $[16,17]$. Despite the availability of newer modalities, the availability and cost issues still make TURP the most frequently performed procedure $[10,18]$. Our study did not find differences in postoperative complication and recatheterization rates between those with and without preoperative Foley catheterization. Although the Qmax was lower in those with the preoperative catheters, they demonstrated higher subjective satisfaction reflected by their lower postoperative IPSS and QOL scores.

Increased adverse perioperative outcomes had been documented in patients in men with urinary retention $[11,12,19,20]$. In our study, the overall complication rate was $16.1 \%$ with the majority being Clavien I (69.5\%) with a higher complication rate in Group 1 than in Group 2 (26.9\% vs $10 \%)$. This was similar to that reported in other studies $[20,21]$. The higher rate of UTI in our study may be due to the increased prevalence of preoperative urine infection in patients with AUR. Our findings are in agreement with other studies reporting a higher rate of infection in those with urinary retention [11,19].

Prolonged duration of surgery and higher volume of resection has been shown to increase complications [19]. In our study, the operation duration was longer with the higher resected volume of the prostate in those with preoperative catheterization. However, there was no significant difference with regard to complications higher than Clavien II. The improvement in complication rate in recent studies can be attributed to the advancements in surgical technique and technology related to the instrument's design $[13,15,22]$.

A transfusion rate of $2 \%$ to $5 \%$ has been reported in various studies [22-25]. Chen et al. reported a higher transfusion rate (3.2\% vs $1.5 \%$ ) and hematuria ( $8.1 \%$ vs $7.4 \%$ ) in patients with AUR than those without AUR [11]. In our study, one patient (1.9\%) required transfusion and two patients (3.8\%) required cystoscopy and clot evacuation in a preoperatively catheterized group of patients while none of the patients without a catheter had hematuria or required transfusion. Sagen et al. also reported higher blood loss in patients with AUR [25]. Catheter-induced cystitis and prostatitis with fragile vessels after prostatic infarction have been implicated in increased blood loss in patients with per-urethral catheters [12,19,20]. 
In our study, TUR syndrome was noted in one patient $(0.7 \%)$ and he recovered uneventfully after two days of management in the intensive care unit. Our findings are in accordance with the review which reported $0 \%$ $1 \%$ incidence of transurethral resection syndrome [24].

A higher proportion of men failing to void after TURP has been documented in those with preoperative urinary catheters $[11,12,17,19,23]$. In our study, recatheterization at the time of discharge was required in 5.7 $\%$ and $3.3 \%$ of patients with and without preoperative catheterization respectively at the time of discharge. All these patients were catheter-free at one week of surgery. A higher recatheterization rate was reported in patients with catheters preoperatively with $23 \%$ of the patients with retention requiring catheters in contrast to only $8 \%$ of those patients without preoperative retention [25]. Chen et al. also reported a $13.8 \%$ recatheterization rate in patients with AUR whereas none of the patients without AUR required so [11]. Similar to our study Johnsen et al. did not find a difference in recatheterization rate regardless of preoperative retention status [14].

In our study, the patients with retention were older with larger prostate and had longer operative duration and greater volume of prostate resected. The mean PSA was higher in a catheterized group ( $3.87 \pm 2.07 \mathrm{vs}$ $2.77 \pm 161 \mathrm{ng} / \mathrm{mL}$ ). Similar findings were documented by Sagen et al. [25]. The higher prostate volume and the Foley catheter-induced prostatic inflammation may have led to raised PSA in this group.

The mean preoperative IPSS and QOL scores in the non-catheterized group were 25.70 and 5.72, respectively, which was similar to the findings by Luitel et al. but higher than reported by Choi et al. $[15,26]$. The higher IPSS score may be due to the delayed presentation of our patients to seek care after the symptoms were significantly bothersome. In this study, the patients with preoperative catheterization had better subjective symptoms scores as reflected in the lower postoperative IPSS and QOL scores. These findings are similar to the study Sagen et al. [25] where patients with AUR had lower IPSS and QOL scores than those without AUR. In contrast to our findings, Chuang et al. [27] did not find a significant difference in the scores between those with and without retention. Getting rid of the urethral catheters may have also contributed to better QOL in those with catheters preoperatively.

Patients with catheters had significantly lower maximum flow rates postoperatively than those without. The cause of AUR in these patients may be the combination of BEP and some degree of detrusor insufficiency which may have affected the Qmax. A similar reduction in flow rate in patients with AUR was reported by Choi et al. [13]. In contrast, Sagen et al. did not find a difference in maximum flow rate between the groups $[25]$.

Patients in our part of the world present late due to financial reasons, a misconception that it is the normal aging process, fear of surgery and need to travel a greater distance to seek a cure and thus need to have a catheter for a longer duration preoperatively $[5,15]$. This may be the reason for the significant number of patients having catheters for more than four weeks preoperatively. Prolonged urinary catheterization has been associated with a significantly higher rate of adverse events such as hematuria, and infection, especially in older patients $[3,12,28]$. We did not find a difference in the mean change in hemoglobin, resected prostate volume and, duration of Foley catheter postoperatively with regards to the various duration of preoperative catheterization. Similarly, Qmax, IPSS and QOL scores at three months were also similar in those subgroups. Increased complications were observed in patients with preoperative Foley catheterization for more than 12 weeks together with the non-significant increased need for recatheterization before discharge. This may be due to the morbidity associated with prolonged catheterization. Similar findings were noted in a study by Das et al. who also failed to find differences in recatheterization rate even in patients with a longer duration of preoperative catheterization [29].

The loss to follow up of a significant number of patients in this sample size makes it the major limitation of the study. Secondly, the duration of follow-up of only three months duration was short to assess long-term post-operative voiding outcomes and complications. The study being a single-centered study limits the generalizability of the results. Transabdominal ultrasound was used to estimate prostate volume instead of transrectal ultrasound which would have provided a better estimation of the prostate volume.

\section{Conclusions}

Our study demonstrated similar postoperative voiding outcomes in patients with preoperative per-urethral catheterization with comparable complication rates to those without catheters. Patients with catheters had better postoperative subjective symptom scores in spite of having a lower postoperative maximum flow rate than those without it. There was no significant difference in complication rate even in those with prolonged catheterization.

These findings have implications for urologists and patients as a significant number of patients still undergo surgery for BPH after suffering an episode of AUR and need to be on an indwelling catheter for a longer duration due to various causes in our part of the world. Previously, these patients were reluctant to undergo surgery as they were counseled regarding inferior postoperative improvements in voiding parameters. The findings of our study may help in counseling these patients as they can expect similar improvement in 
voiding parameters as those without catheters. Further clinical studies with large sample size and longer follow-up period are warranted to validate these findings.

\section{Additional Information \\ Disclosures}

Human subjects: Consent was obtained or waived by all participants in this study. Institutional Review Board of National Academy of Medical Sciences issued approval 529/77/78. Ethical clearance was obtained from the Institutional Review Board of the National Academy of Medical Sciences. Animal subjects: All authors have confirmed that this study did not involve animal subjects or tissue. Conflicts of interest: In compliance with the ICMJE uniform disclosure form, all authors declare the following: Payment/services info: All authors have declared that no financial support was received from any organization for the submitted work. Financial relationships: All authors have declared that they have no financial relationships at present or within the previous three years with any organizations that might have an interest in the submitted work. Other relationships: All authors have declared that there are no other relationships or activities that could appear to have influenced the submitted work.

\section{References}

1. Wei JT, Calhoun E, Jacobsen SJ: Urologic diseases in America project: benign prostatic hyperplasia . J Urol. 2005, 173:1256-61. 10.1097/01.ju.0000155709.37840.fe

2. Egan KB: The epidemiology of benign prostatic hyperplasia associated with lower urinary tract symptoms: prevalence and incident rates. Urol Clin North Am. 2016, 43:289-97. 10.1016/j.ucl.2016.04.001

3. Fitzpatrick JM, Desgrandchamps F, Adjali K, et al.: Management of acute urinary retention: a worldwide survey of 6074 men with benign prostatic hyperplasia. BJU Int. 2012, 109:88-95. 10.1111/j.1464410X.2011.10430.x

4. Izard J, Nickel JC: Impact of medical therapy on transurethral resection of the prostate: two decades of change. BJU Int. 2011, 108:89-93. 10.1111/j.1464-410X.2010.09737.X

5. Loh SY, Chin CM: A demographic profile of patients undergoing transurethral resection of the prostate for benign prostate hyperplasia and presenting in acute urinary retention. BJU Int. 2002, 89:531-3. 10.1046/j.1464-410x.2002.02662.x

6. Cornu JN, Ahyai S, Bachmann A, et al.: A systematic review and meta-analysis of functional outcomes and complications following transurethral procedures for lower urinary tract symptoms resulting from benign prostatic obstruction: an update. Eur Urol. 2015, 67:1066-96. 10.1016/j.eururo.2014.06.017

7. Foster HE, Dahm P, Kohler TS, et al.: Surgical management of lower urinary tract symptoms attributed to benign prostatic hyperplasia: AUA guideline amendment. J Urol. 2019, 202:592-8. $10.1097 /$ ju.0000000000000319

8. EAU guidelines. (2021). Accessed: May 3, 2021: http://www.uroweb.org/guideline/treatment of nonneurogenic-male-luts/.

9. Huang LK, Chang YH, Shao IH, et al.: Clinical outcome of immediate transurethral surgery for benign prostate obstruction patients with acute urinary retention: more radical resection resulted in better voiding function. J Clin Med. 2019, 8:1278. 10.3390/jcm8091278

10. Morton A, Williams M, Perera M, et al.: Management of benign prostatic hyperplasia in the 21st century: temporal trends in Australian population-based data. BJU Int. 2020, 126:18-26. 10.1111/bju.15098

11. Chen JS, Chang CH, Yang WH, et al.: Acute urinary retention increases the risk of complications after transurethral resection of the prostate: a population-based study. BJU Int. 2012, 110:E896-901. 10.1111/.1464-410X.2012.11471.x

12. Pickard R, Emberton M, Neal DE: The management of men with acute urinary retention . Br J Urol. 1998, 81:712-20. 10.1046/j.1464-410x.1998.00632.x

13. Choi HS, Kim DJ, Kim DS, et al.: Factors affecting the improvement of the initial peak urinary flow rate after transurethral resection of the prostate or photoselective vaporization of the prostate for treating benign prostatic hyperplasia. Int Neurourol J. 2011, 15:35-40. 10.5213/inj.2011.15.1.35

14. Johnsen NV, Kammann TJ, Marien T, Pickens RB, Miller NL: Comparison of holmium laser prostate enucleation outcomes in patients with or without preoperative urinary retention. J Urol. 2016, 195:1021-6. 10.1016/j.juro.2015.10.116

15. Luitel B, Gupta D, Chalise P, et al.: Change in storage symptoms after transurethral resection of prostate: a prospective observational study. J Soc Surgeons Nepal. 2016, 17:35-8. 10.3126/issn.v17i1.15179

16. Choi SY, Kim TH, Myung SC, et al.: Impact of changing trends in medical therapy on surgery for benign prostatic hyperplasia over two decades. Korean J Urol. 2012, 53:23-8. 10.4111/kju.2012.53.1.23

17. Khan M, Khan AL, Khan S, et al.: Benign prostatic hyperplasia: mode of presentation and postoperative outcome. J Pak Med Assoc. 2005, 55:20-3.

18. Jeon BJ, Chung H, Bae JH, et al.: Analysis of present status for surgery of benign prostatic hyperplasia in Korea using nationwide healthcare system data. Int Neurourol J. 2019, 23:22-9. 10.5213/inj.1836198.099

19. Mebust WK, Holtgrewe HL, Cockett AT, et al.: Transurethral prostatectomy: immediate and postoperative complications. A cooperative study of 13 participating institutions evaluating 3,885 patients. J Urol. 1989, 141:243-7. 10.1016/s0022-5347(17)40731-2

20. Reich O, Gratzke C, Bachmann A, et al.: Morbidity, mortality and early outcome of transurethral resection of the prostate: a prospective multicenter evaluation of 10,654 patients. J Urol. 2008, 180:246-9. 10.1016/.j.juro.2008.03.058

21. Guo R, Yu W, Meng Y, et al.: Correlation of ASA grade and the Charlson comorbidity index with complications in patients after transurethral resection of postate. Urology. 2016, 98:120-5. 10.1016/j.urology.2016.07.025 


\section{Cureus}

22. Guo RQ, Yu W, Meng YS, et al.: A nomogram predicting re-operation due to secondary hemorrhage after monopolar transurethral resection of prostate. Kaohsiung J Med Sci. 2018, 34:172-8.

10.1016/j.kjms.2017.08.008

23. Muntener M, Aellig S, Kuttel R, et al.: Peri-operative morbidity and changes in symptom scores after transurethral prostatectomy in Switzerland: results of an independent assessment of outcome. BJU Int. 2006, 98:381-3. 10.1111/j.1464-410X.2006.06256.x

24. Rassweiler J, Teber D, Kuntz R, et al.: Complications of transurethral resection of the prostate (TURP)-incidence, management, and prevention. Eur Urol. 2006, 50:969-79. 10.1016/j.eururo.2005.12.042

25. Sagen E, Hedelin H, Nelzen O, et al.: Defining and discriminating responders from non-responders following transurethral resection of the prostate. Scand J Urol. 2018, 52:437-44. 10.1080/21681805.2018.1536677

26. Choi H, Kim JH, Shim JS, et al.: Prediction of persistent storage symptoms after transurethral resection of the prostate in patients with benign prostatic enlargement. Urol Int. 2014, 93:425-30. 10.1159/000357626

27. Chuang FP, Lee SS, Wu ST, et al.: Change in International Prostate Symptom Score after transurethral prostatectomy in Taiwanese men with benign prostate hyperplasia: use of these changes to predict the outcome. Arch Androl. 2003, 49:129-37. 10.1080/01480510390129250

28. Emberton M, Zinner N, Michel MC, et al.: Managing the progression of lower urinary tract symptoms/benign prostatic hyperplasia: therapeutic options for the man at risk. BJU Int. 2007, 100:249-53. 10.1111/j.1464410X.2007.07056.x

29. Das Bhagia S, Mahmud SM, El Khalid S: Is it necessary to remove foleys catheter late after transurethral prostatectomy in patients who presented with acute urinary retention secondary to benign prostatic hyperplasia?. J Pak Med Assoc. 2010, 60:739-41. 